\title{
Research
}

Christopher C Butler, Jonathan AC Sterne, Michael Lawton, Kathryn O’Brien, Mandy Wootton, Kerenza Hood, William Hollingworth, Paul Little, Brendan C Delaney, Judith van der Voort, Jan Dudley, Kate Birnie, Timothy Pickles, Cherry-Ann Waldron, Harriet Downing, Emma Thomas-Jones, Catherine Lisles, Kate Rumsby, Stevo Durbaba, Penny Whiting, Kim Harman, Robin Howe, Alasdair MacGowan, Margaret Fletcher and Alastair D Hay

\section{Nappy pad urine samples for investigation and treatment of UTI in young children:}

\author{
the 'DUTY' prospective diagnostic cohort study
}

\begin{abstract}
Background

The added diagnostic utility of nappy pad urine samples and the proportion that are contaminated is unknown.
\end{abstract}

Aim

To develop a clinical prediction rule for the diagnosis of urinary tract infection (UTI) based on sampling using the nappy pad method.

\section{Design and setting}

Acutely unwell children $<5$ years presenting to 233 UK primary care sites.

\section{Method}

Logistic regression to identify independent associations of symptoms, signs, and urine dipstick test results with UTI; diagnostic utility quantified as area under the receiver operator curves (AUROC). Nappy pad rule characteristics, AUROC, and contamination, compared with findings from clean-catch samples.

\section{Results}

Nappy pad samples were obtained from 3205 children ( $82 \%$ aged $<2$ years; $48 \%$ female), culture results were available for $2277(71.0 \%)$ and $30(1.3 \%)$ had a UTI on culture. Female sex smelly urine, darker urine, and the absence of nappy rash were independently associated with a UTI, with an internally-validated, coefficient model AUROC of 0.81 (0.87 for clean-catch), which increased to 0.87 ( 0.90 for clean-catch) with the addition of dipstick results. GPs' 'working diagnosis' had an AUROC 0.63 (95\% confidence intervals $[\mathrm{Cl}]=0.53$ to 0.72 ). A total of $12.2 \%$ of nappy pad and $1.8 \%$ of cleancatch samples were 'frankly contaminated' (risk ratio $6.66 ; 95 \% \mathrm{Cl}=4.95$ to 8.96 ; $P<0.001$ )

\section{Conclusion}

Nappy pad urine culture results, with features that can be reported by parents and dipstick tests, can be clinically useful, but are less accurate and more often contaminated compared with clean-catch urine culture.

\section{Keywords}

antibacterial agents; diagnosis; infant; paediatrics; primary health care; urinary tract infections.

\section{INTRODUCTION}

Urinary tract infection (UTI) may be missed in up to $80 \%$ of children presenting to primary care ${ }^{1,2}$ Accurate diagnosis of UTI is essential to avoid over- or undertreatment with antibiotics and to appropriately target burdensome and expensive investigations. ${ }^{3}$ This is especially important in younger, pre-verbal children who are not yet toilettrained and who often present with nonspecific symptoms, making the decision about which children to investigate for UTI difficult. $^{3}$ Obtaining a urine sample can be time consuming and especially challenging in primary care, where most children first present. ${ }^{4}$ The nappy pad sampling method in young children in nappies (diapers), when

CC Butler, FMedSci, professor of primary care, Nuffield Department of Primary Care Health

Sciences, University of Oxford, Radcliffe Observatory Quarter, Oxford, and GP, Cwm Taf University Health Board. JAC Sterne, PhD, professor of medical statistics and epidemiology; M Lawton MPhil, research associate; K Birnie, PhD, research fellow; W Hollingworth, PhD, professor of health economics, School of Social and Community Medicine, University of Bristol, Bristol. K O'Brien, $\mathrm{PhD}$, clinical lecturer, Division of Population

Medicine, School of Medicine, Cardiff University, Cardiff. M Wootton, PhD, lead scientist; R Howe, FRCPath, lead scientist, Specialist Antimicrobial Chemotherapy Unit, Public Health Wales Microbiology Cardiff, University Hospital Wales, Cardiff. K Hood, PhD, director; T Pickles, MSc, statistician; C-A Waldron, PhD, trial manager; E Thomas-Jones, PhD, research fellow; C Lisles MSc, data manager, South East Wales Trials Unit (SEWTU), Centre for Trials Research, Cardiff University, Cardiff. P Little, FMedSci, professor of primary care research; K Rumsby, MSc, trial manager; K Harman, Dhealth, trial manager, Primary Care and Population Sciences Division University of Southampton, Southampton. BC Delaney, MD, chair in medical informatics and decision making. Department of Surgery and Cancer, Imperial College, St Mary's Hospital, London. J van der Voort, FRCPCH, consultant paediatrician, Department of Paediatrics and Child Health, University Hospital of Wales, Cardiff. J Dudley, $\mathrm{PhD}$, consultant paediatric nephrologist, Bristol Royal Hospital for Children, University a clean-catch sample cannot be obtained, has been recommended by the National Institute for Health and Care Excellence (NICE). ${ }^{3}$ Urine sampling needs to be simple, reliable, and acceptable, and parents find nappy pads easy to use, comfortable, for their children and prefer them to the cleancatch method..$^{5}$ Nappy pad sampling is used in everyday care, ${ }^{1}$ and GPs report using nappy pad urine collection in over $40 \%$ of infants. ${ }^{6}$ Many parents feel that the cleancatch method is messy and time consuming and give up trying. ${ }^{3,5}$ However, the clinical utility of the information obtained from the nappy pad method of urine sampling is unclear, contamination rates may be higher than other sampling methods, and

Hospitals Bristol, NHS Foundation Trust, Bristol. H Downing. MPhil, trial manager; AD Hay, FRCGP, professor of primary care, Centre for Academic Primary Care, NIHR School of Primary Care Research, School of Social and Community Medicine, University of Bristol, Bristol. S Durbaba MSc, e-resources developer, King's College London, Division of Health and Social Care Research, Department of Primary Care and Public Health Sciences, London. P Whiting, PhD, senior research fellow, NIHR CLAHRC West, University Hospitals Bristol NHS Foundation Trust, Bristol. A MacGowan, MD, professor of clinical microbiology and antimicrobial therapeutics, North Bristol NHS Trust, Southmead Hospital, Bristol. M Fletcher, $\mathrm{PhD}$, formerly professor of clinical nursing, University of the West of England, Bristol with University Hospitals Bristol, NHS Foundation Trust, Bristol.

\section{Address for correspondence}

Christopher C Butler, Nuffield Department of Primary Care Health Sciences, University of Oxford, Radcliffe Primary Care Building, Radcliffe Observatory Quarter, Woodstock Road, Oxford OX2 6GG, UK.

E-mail: christopher.butleraphc.ox.ac.uk

Submitted: 10 December 2015; Editor's response: 2 January 2016; final acceptance: 25 February 2016. CBritish Journal of General Practice

This is the full-length article (published online 1 Jul 2016) of an abridged version published in print. Cite this version as: Br J Gen Pract 2016; DOI: 10.3399/bjgp16X685873 


\section{How this fits in}

Up to $80 \%$ of urinary tract infections (UTI) in young children presenting to primary care are missed. Timely and accurate diagnosis is essential to avoid over- or undertreatment and investigation. This is especially difficult in pre-verbal children who are not toilet trained, and present with undifferentiated symptoms. GPs use, and parents prefer, nappy pads for collecting urine from children who are still in nappies, but the clinical utility of data derived from nappy pad samples, the added value of dipstick testing, and the proportion of contaminated samples is not known. It was found that culture results from urine obtained using nappy pads, together with features that can be reported by parents, can be clinically useful in identifying acutely unwell pre-school children presenting to primary care who have a UTI, but with less accuracy compared to clean-catch sampling. However, contamination rates are nearly seven times higher in nappy pads than in clean-catch samples. Cleancatch urine sampling in children in primary care should therefore be prioritised over the nappy pad method, but if urine sampling is done using nappy pads, then the addition of dipstick testing significantly improves diagnostic accuracy.

children in nappies present differently to older children who are more able to describe symptoms and in whom cleancatch sampling is easier. Obtaining urine samples by more invasive methods such as suprapubic aspiration or catheterisation is neither feasible nor acceptable in most primary care settings.

The aim of the study therefore was to develop a clinical prediction rule for the diagnosis of UTI based on sampling using the nappy pad method, and compare its diagnostic utility to a similar rule based on 'clean-catch' urine samples.? In addition, the added diagnostic value of dipstick testing once a nappy pad sample had been obtained was estimated, and contamination rates were compared by the sampling method.

\section{METHOD \\ Participants}

The Diagnosis of Urinary Tract infection in Young children (DUTY) study was a multicentre, prospective, diagnostic cohort study that recruited children aged $<5$ years, in primary care. ${ }^{8}$ Children were eligible if presenting with any acute ( $<28$ days), undifferentiated illness leven when the clinician was confident of the diagnosis, such as a child with bronchiolitis), and/or new urinary symptoms.

\section{Index tests and urine collection}

Following consent, 107 index test items were recorded. Parent-reported items included the child's medical history and symptoms. Clinician-assessed items came from a full clinical examination, including their global impression of illness severity (rated $0-10$ ), their rating of the likelihood of UTI, and urine dipstick results (performed after rating UTI likelihood). Index test items were derived from a literature review and input from the co-investigator group.

The NICE recommended 'Newcastle Nappy Pads' were used for those children who wore nappies (diapers) and for those in whom the parent/guardian did not think clean-catch would be successful., 3,8 First, the parent was asked to clean the nappy area using water or wipes (the wipes being supplied by the study). A nappy pad was inserted inside a clean nappy, and the nappy refastened. The nappy pad was removed as soon as the child urinated, in order to reduce the risk of contamination. The perineum was cleaned again and a fresh pad inserted every 30 minutes until micturition, or immediately if the pad became contaminated with faeces. Once the child had urinated, the research nurse or clinical study officer (RN/ CSO), wearing disposable gloves, removed the pad and urine was extracted into a sterile container as per the manufacturer's instructions (Newcastle Urine Collection Pack; Ontex Ltd, Corby, UK; NHS Supplies). If it was not possible to obtain a sample before the child left the primary care site, the parent was given the necessary equipment and advice on obtaining a urine sample at home. The parent was advised to store the sample in the fridge and return it to their primary care site as soon as possible, ideally within 24 hours. The RN/CSO telephoned parents the next day to remind them to return the sample. Where feasible, the RN/ CSO offered to collect the urine sample from the child's home.

At the primary care site, urine samples were dipstick tested lusing Siemens/Bayer Multistix 8SG) for blood, protein, glucose, ketones, nitrite, leukocyte esterase, $\mathrm{pH}$, and specific gravity leight dipstick index tests). All index tests and the clinician's working diagnosis ('clinical diagnosis') were measured blind to the reference standard.

When there was at least $1 \mathrm{~mL}$ of urine leftover after the priority sample was sent to a NHS laboratory, it was sent, in boric acid monovettes, using first class Royal Mail Safeboxes $^{T M}$, to the Public Health Wales 
Microbiology NHS Laboratory in Cardiff la research laboratory). Results from the research laboratory are used in the current analyses.

\section{Reference standard}

The research laboratory spiral-plated (Don Whitley Scientific, Shipley, UKJ $50 \mu \mathrm{L}$ onto chromogenic agar and standard blood agar. A full description of the methods used in the research laboratory, and how these differed from the range of standard operating procedures in local NHS laboratories, is forthcoming.10 Quantitative total counts were recorded for up to six organisms and the presence of antimicrobial substances measured. Samples were processed using a single, standardised procedure. Uropathogens were defined as members of the Enterobacteriaceae group. The microbiological definition of UTI used was the presence of $\geq 10^{5}$ colony-forming units (CFU)/mL of a single uropathogen ('pure growth'), or $\geq 10^{5} \mathrm{CFU} / \mathrm{mL}$ of a uropathogen with $a \geq 3 \log _{10}$ (1000-fold) difference between the growth of this and the next species ('predominant growth'). Aside from the children's dates of birth, laboratory staff were blind to index tests. As there is no single accepted definition of contamination, three definitions were considered: growth of $>2$ organisms of $>10^{5} \mathrm{CFU} / \mathrm{mL}$ l'frank contamination'); ${ }^{11}$ growth of $\geq 2$ organisms at $>10^{5} \mathrm{CFU} / \mathrm{mL}$ ("heavy contamination' according to Rao et $a l^{l^{2}}$ and Feasey $\left.{ }^{13}\right)$, and growth of $>2$ organisms at $>10^{4} \mathrm{CFU} / \mathrm{mL}$ 'probable contamination' according to Jackson et $a l^{(1)}$, or 'frank contamination' according to Bekeris et a ${ }^{(4)}$.

\section{Statistical analysis}

The frequency of symptom and sign categories were examined, blind to their associations with urine culture results, and the least frequent categories were merged before analyses. Logistic regression was used to estimate associations of index tests with urine culture positivity. $P$-values were derived using likelihood ratio tests. For ordinal variables, both heterogeneity and trend $P$-values were derived. Continuous variables were divided into quintiles and trend $P$-values were derived using the median within categories. Plots of the log odds of culture positivity were examined against the median within quintiles for evidence of non-linearity.

Two methods for dealing with missing data were used, including the response 'don't know' to questions about the presence of symptoms such as pain or crying, when passing urine. In both univariable and multivariable analyses missing data were coded as the modal value, usually as absence of the symptom. Multivariable analyses were repeated using the chained equations approach to multiple imputation: estimates and Wald $P$-values ${ }^{15}$ based on 50 imputed datasets derived using Rubin's rules. ${ }^{16}$

Prediction rules were derived in three stages. First, symptoms and signs were selected, with either trend or heterogeneity univariable $P$-values $<0.01$. Second, models were derived from selected symptoms and signs using backwards stepwise selection and an exclusion criterion of heterogeneity $P$-value $>0.1$. Third, backwards stepwise selection was used, with the same exclusion $P$-value for models in which dipstick results were added. The effect of using more liberal $P$-value thresholds of 0.1 and 0.2 at the first stage was investigated, and no important differences were found in the final models (results available from the authors on request).

Diagnostic accuracy was quantified as the area under the receiver operating characteristic (AUROC) curve lalso known as the 'c-statistic'). AUROC values for clinical judgement of UTI were also estimated. Internal validation of the models was conducted using the bootstrap procedure described by Steyerberg ${ }^{17}$ and a calibration slope (shrinkage factor) was calculated, by which model coefficients were multiplied, in order to derive internallyvalidated odds ratios (OR). For each model, cut-points corresponding to a range of values for sensitivity were selected, and then the corresponding specificity, negative and positive predictive values, and the proportion of children classified positive, were calculated. These were compared against 'clinical diagnosis' of UTI lwhere clinicians considered UTI to be 'fairly' or 'very' certain). Models were re-run, leaving out predictive features that could lack face validity.

\section{Sample size calculation}

The sample size calculation assumed a candidate predictor with $10 \%$ prevalence and UTI prevalence of $2 \%$. With $80 \%$ power and a two-sided $\alpha$ of $5 \%, 3000$ urine sample results were required to detect an OR of 2.4 , while 3100 results would give a $95 \%$ confidence interval $[\mathrm{Cl}]$ with width $10 \%$ for an algorithm with $80 \%$ sensitivity. It was originally proposed to recruit 4000 children, combining analyses for children with both clean-catch and nappy pad samples, anticipating recovery of urine samples from $77.5 \%$ for algorithm derivation, and a 


\section{Table 1. Nappy pad samples: children's characteristics and crude odds ratios for index tests associated with UTI}

\begin{tabular}{|c|c|c|c|c|}
\hline Demographics/index tests & $N(\%)^{a}$ & UTI (\%) $)^{b}$ & Crude $\mathbf{O R}^{\mathrm{c}}$ & $95 \% \mathrm{Cl}$ \\
\hline Total & 2277 & $30(1.3)$ & & \\
\hline \multicolumn{5}{|l|}{ Age of child } \\
\hline$<6$ months & $369(16.2)$ & $5(1.4)$ & 1.72 & 0.54 to 5.46 \\
\hline 6 to $<12$ months & $603(26.5)$ & $11(1.8)$ & 2.33 & 0.90 to 6.04 \\
\hline 1 to $<2$ years & $884(38.8)$ & $7(0.8)$ & 1 & ref \\
\hline 2 to $<3$ years & $353(15.5)$ & $7(2.0)$ & 2.53 & 0.88 to 7.28 \\
\hline 3 to $<4$ years & $58(2.5)$ & $0(0.0)$ & $\mathrm{n} / \mathrm{a}$ & \\
\hline$\geq 4$ years & $10(0.4)$ & $0(0.0)$ & $\mathrm{n} / \mathrm{a}$ & \\
\hline \multicolumn{5}{|c|}{ Time from index tests to taking urine sample } \\
\hline Sample before recruitment & 120 (5.3) & $2(1.7)$ & 1.33 & 0.31 to 5.67 \\
\hline$<24$ hours & 1982 (87.0) & 25 (1.3) & 1 & ref \\
\hline 24 to $<48$ hours & 109 (4.8) & $3(2.8)$ & 2.22 & 0.66 to 7.45 \\
\hline 48 to $<72$ hours & $18(0.8)$ & $0(0.0)$ & $\mathrm{n} / \mathrm{a}$ & \\
\hline$\geq 72$ hours & $48(2.1)$ & $0(0.0)$ & $\mathrm{n} / \mathrm{a}$ & \\
\hline \multicolumn{5}{|l|}{ Clinician diagnosis prior to dipstick } \\
\hline No UTI certain to very certain & $1033(45.4)$ & $8(0.8)$ & 0.52 & 0.22 to 1.19 \\
\hline Uncertain or no UTI fairly certain & $1201(52.7)$ & $18(1.5)$ & 1 & ref \\
\hline UTI fairly to very certain & $38(1.7)$ & $4(10.5)$ & 7.76 & 2.49 to 24.18 \\
\hline Missing & $5(0.2)$ & $0(0.0)$ & & \\
\hline \multicolumn{5}{|l|}{ Sex } \\
\hline Male & $1183(52.0)$ & $9(0.8)$ & 1 & ref \\
\hline Female & 1094 (48.0) & $21(1.9)$ & 2.55 & 1.16 to 5.60 \\
\hline \multicolumn{5}{|l|}{ Smelly urine } \\
\hline No problem & $1518(66.7)$ & $12(0.8)$ & 1 & ref \\
\hline Slight problem & $206(9.0)$ & $4(1.9)$ & 2.20 & 0.73 to 6.61 \\
\hline Moderate problem & $138(6.1)$ & $5(3.6)$ & 4.18 & 1.52 to 11.50 \\
\hline Severe problem & $26(1.1)$ & $4(15.4)$ & 20.21 & 6.29 to 64.97 \\
\hline Missing/not known & $389(17.1)$ & $5(1.3)$ & & \\
\hline \multicolumn{5}{|l|}{ Darker urine } \\
\hline No problem & 1764 (77.5) & $19(1.1)$ & 1 & ref \\
\hline Slight problem & $83(3.6)$ & $2(2.4)$ & 2.19 & 0.51 to 9.43 \\
\hline Moderate or severe problem & $41(1.8)$ & 4 (9.8) & 9.59 & 3.17 to 29.02 \\
\hline Missing/not known & $389(17.1)$ & $5(1.3)$ & & \\
\hline \multicolumn{5}{|l|}{ Nappy rash ${ }^{d}$} \\
\hline No problem & 1715 (75.3) & $29(1.7)$ & 1 & ref \\
\hline Slight to severe problem & $560(24.6)$ & $1(0.2)$ & 0.10 & 0.01 to 0.77 \\
\hline Missing & $2(0.1)$ & $0(0.0)$ & & \\
\hline \multicolumn{5}{|l|}{ Dipstick: leucocytes } \\
\hline Negative & 1759 (77.3) & $13(0.7)$ & 1 & ref \\
\hline Trace & $125(5.5)$ & $1(0.8)$ & 1.09 & 0.14 to 8.38 \\
\hline+ & 119 (5.2) & 4 (3.4) & 4.69 & 1.50 to 14.61 \\
\hline++ & $177(7.8)$ & $4(2.3)$ & 3.12 & 1.01 to 9.66 \\
\hline+++ & $91(4.0)$ & 8 (8.8) & 12.99 & 5.24 to 32.20 \\
\hline Missing & $6(0.3)$ & $0(0.0)$ & & \\
\hline \multicolumn{5}{|l|}{ Dipstick: nitrites } \\
\hline Negative & $1916(84.1)$ & $13(0.7)$ & 1 & ref \\
\hline Positive & $355(15.6)$ & $17(4.8)$ & 7.39 & 3.55 to 15.35 \\
\hline Missing & $6(0.3)$ & $0(0.0)$ & & \\
\hline
\end{tabular}

$O R=$ odds ratio. $r$ ref $=$ reference. $U T I=$ urinary tract infection. ${ }^{a}$ Percentage relative to the total number of observations ( $N=2277$ ). ${ }^{b}$ Percentage relative to the total number of observations within that category. ${ }^{c} \mathrm{Crude}$ ORs calculated using modal imputation. nappy pad results to derive the model, and to conduct internal bootstrap validation instead of external validation.

\section{RESULTS}

\section{Participants}

Participants from 233 primary care sites (225 GP practices, four walk-in centres and four children's emergency departments [EDs]) across England and Wales between April 2010 and April 2012, were recruited. Of 10138 children who screened eligible, 1276 (12.6\%) declined participation, 1684 (16.6\%) could not be recruited for other reasons, and $15(0.15 \%)$ withdrew.

Of the 233 primary care sites taking part, 198 sites (85\%) completed and returned at least one screening log to the study centre. These showed that 7350 children were screened but not recruited because they declined $(n=1276)$, were not eligible ( $n=4390)$, or for other reasons ( $n=1684)$, which included: left the primary care site prior to invitation $(n=811)$; did not give consent ( $n=214)$; or there was a language barrier $(n=112)$ and an appropriate translator was not available at the time of recruitment. There were slightly more males Imean difference of $5.2 \% ; 95 \%=\mathrm{Cl} 2.2$ to $8.2 \%$ l among those for whom participation was declined $(n=1276)$ compared with those who did agree to participate in the DUTY study ( $n=7163)$. The mean age in the declined sample was 24.06 months versus 26.88 months among participants Imean difference 2.04 months, $95 \% \mathrm{Cl}=1.08$ to 3.00 months). Clinical information on those who declined was not collected.

Urine was collected from a total of 6241 children, 3205 using nappy pads. A total of 3164 (98.7\%) nappy pad samples were sent to NHS laboratories, and 2363 (73.7\%) to the research laboratory. The number of reference standard results available from the research laboratory (the final analytical sample) was 2277 (71.0\%). A total of $82 \%$ of children providing nappy pad samples were aged <2 years (mean 1.3 years, SD 0.8), and the mean illness severity score was 2.3 points (SD 1.5); $48 \%$ of children were female; and overall, $1.3 \%$ had a UTI (Table 1). The clean-catch sample $(n=2740)$ had a mean age of 3.5 years (SD 1.0), mean illness severity score of 2.2 points (SD 1.6), and $53.8 \%$ were female.

In total, 2102 (92.3\%) samples were provided within 24 hours of the index test measurement, and there was no relationship between UTI and time from urine collection to laboratory arrival. Antimicrobial substances, which can arise from the use of both systemic antibiotics and 


\section{Table 2. Nappy pad samples: models based on symptoms and signs; and on symptoms, signs, and dipstick}

results, including results based on multiple imputation

\begin{tabular}{|c|c|c|c|c|c|c|c|c|}
\hline \multirow[b]{3}{*}{ Index tests } & \multicolumn{4}{|c|}{ Symptom and sign model } & \multicolumn{4}{|c|}{ Symptom sign and dipstick model } \\
\hline & \multirow{2}{*}{$\begin{array}{l}\text { Adjusted } \\
\text { OR }^{\mathrm{a}}\end{array}$} & \multicolumn{3}{|c|}{ MI } & \multirow{2}{*}{$\begin{array}{l}\text { Adjusted } \\
\text { OR }\end{array}$} & \multicolumn{3}{|c|}{ MI } \\
\hline & & $95 \% \mathrm{Cl}^{\mathrm{a}}$ & adjusted OR & $95 \% \mathrm{Cl}$ & & $95 \% \mathrm{Cl}^{\mathrm{a}}$ & adjusted $\mathrm{OR}^{\mathrm{a}}$ & $95 \% \mathrm{Cl}$ \\
\hline \multicolumn{9}{|l|}{ Sex } \\
\hline Male & 1 & ref & 1 & ref & 1 & ref & 1 & ref \\
\hline Female & 1.95 & 1.11 to 3.41 & 1.96 & 1.06 to 3.61 & 1.41 & 0.80 to 2.48 & 1.45 & 0.78 to 2.72 \\
\hline \multicolumn{9}{|l|}{ Smelly urine } \\
\hline No problem & 1 & ref & 1 & ref & 1 & ref & 1 & ref \\
\hline Slight problem & 1.61 & 0.73 to 3.54 & 1.97 & 0.82 to 4.71 & 1.44 & 0.67 to 3.11 & 1.79 & 0.76 to 4.23 \\
\hline Moderate problem & 2.51 & 1.14 to 5.51 & 3.39 & 1.46 to 7.89 & 2.15 & 0.98 to 4.68 & 2.96 & 1.26 to 6.97 \\
\hline Severe problem & 7.40 & 2.98 to 18.36 & 10.14 & 3.85 to 26.69 & 3.97 & 1.58 to 9.96 & 6.13 & 2.28 to 16.47 \\
\hline \multicolumn{9}{|l|}{ Darker urine } \\
\hline No problem & 1 & ref & 1 & ref & 1 & ref & 1 & ref \\
\hline Slight problem & 1.89 & 0.66 to 5.46 & 1.99 & 0.65 to 6.12 & 1.81 & 0.65 to 5.07 & 1.92 & 0.63 to 5.88 \\
\hline Moderate or severe problem & 2.46 & 0.98 to 6.21 & 2.26 & 0.85 to 6.01 & 2.29 & 0.93 to 5.62 & 2.27 & 0.87 to 5.93 \\
\hline \multicolumn{9}{|l|}{ Nappy rash } \\
\hline No problem & 1 & ref & 1 & ref & 1 & ref & 1 & ref \\
\hline Slight to severe problem & 0.16 & 0.04 to 0.66 & 0.13 & 0.03 to 0.61 & 0.19 & 0.05 to 0.71 & 0.16 & 0.04 to 0.66 \\
\hline \multicolumn{9}{|l|}{ Dipstick: leukocytes } \\
\hline Negative & & & & & 1 & ref & 1 & ref \\
\hline Trace & & & & & 0.87 & 0.23 to 3.31 & 0.81 & 0.18 to 3.61 \\
\hline+ & & & & & 2.06 & 0.92 to 4.63 & 2.18 & 0.88 to 5.43 \\
\hline ++ & & & & & 1.63 & 0.73 to 3.62 & 1.78 & 0.73 to 4.30 \\
\hline+++ & & & & & 3.27 & 1.66 to 6.41 & 3.35 & 1.57 to 7.15 \\
\hline \multicolumn{9}{|l|}{ Dipstick: nitrites } \\
\hline Negative & & & & & 1 & ref & 1 & ref \\
\hline Positive & & & & & 3.16 & 1.91 to 5.24 & 3.70 & 2.10 to 6.52 \\
\hline \multicolumn{9}{|c|}{ Receiver operating characteristic curve } \\
\hline ROC & 0.769 & 0.68 to 0.85 & 0.805 & 0.72 to 0.89 & 0.858 & 0.79 to 0.93 & 0.870 & 0.80 to 0.94 \\
\hline Validated ROC ${ }^{b}$ & 0.744 & & 0.778 & & 0.799 & & 0.821 & \\
\hline$\triangle R^{\prime} O C^{c}$ & & & & & 0.089 & 0.02 to 0.16 & 0.065 & 0.00 to 0.13 \\
\hline$\triangle \mathrm{ROC}^{c} P$-value & & & & & 0.012 & & 0.036 & \\
\hline Calibration slope & 0.695 & & 0.749 & & 0.647 & & 0.708 & \\
\hline
\end{tabular}

$O R=$ odds ratio. $M I=$ multiple imputation. $r e f=$ reference. $R O C=$ receiver operating characteristic. ${ }^{a}$ Missing values coded to modal category. ${ }^{b} /$ ternal validation using the bootstrap procedure. The difference in ROC between symptom and sign model and symptom, sign and dipstick model. OR calculated using shrunken estimates from the bootstrap internal validation calibration slope.

Figure 1. Receiver operating characteristic curves for symptoms and signs model (solid line), and symptoms, signs and dipstick model (dotted line) for nappy pad urine samples for diagnosing UTI in young children.

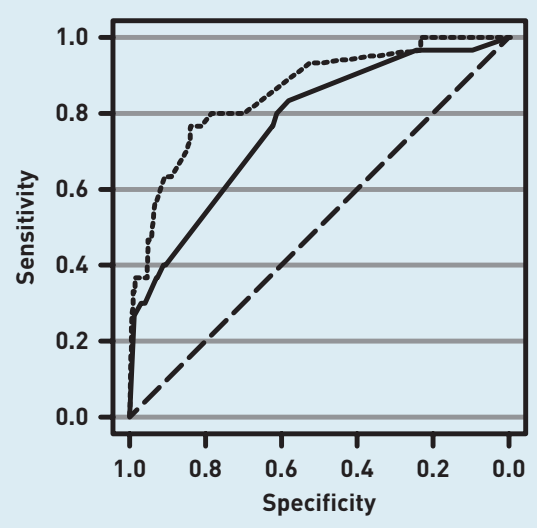

locally applied cleaning agents, were found in $6.6 \%$ of nappy pad samples, and were more likely to be present in children with, than without, UTI. GPs' 'working diagnosis' would have correctly identified four (13.3\%) of the $30 \mathrm{UTI}$, with $97.0 \%$ specificity and an AUROC $0.63(95 \% \mathrm{Cl}=0.53$ to 0.72$)$.

\section{Nappy pad model}

Table 2 shows adjusted ORs for the index tests (Table 1) selected for the nappy pad model. Parent-reported smelly urine, darker urine, female sex, and the absence of a nappy rash, were independently associated with UTI: for the first two, there was evidence of graded associations. No clinical examination findings were independently associated with UTI. The presence of leukocytes and nitrites from dipstick urine testing were independently associated with
UTI. The symptoms and signs model had reasonable diagnostic accuracy lvalidated AUROC for the multiple imputed model was 0.78 and diagnostic accuracy increased $[P=0.036]$ with addition of dipstick findings [validated AUROC 0.82]). Figure 1 shows the multiple imputed receiver operating characteristic curves for the models with and without dipstick urinalysis.

The multiple imputation analysis was re-run, excluding the nappy rash variable, and it was found that there was a reduction of 0.07 in validated AUROC in the symptom and sign model (from 0.78 to 0.71), and a reduction of 0.03 in the validated AUROC in the symptom, sign, and dipstick model (from 0.82 to 0.80 ). The association between antimicrobial substances in the urine and nappy rash was checked, and no association was found $(P=0.82)$. 
Table 3. Numbers of contaminated samples using different definitions of contamination

\begin{tabular}{|c|c|c|c|c|c|c|}
\hline Study & $\begin{array}{l}\text { Contamination } \\
\text { definition }\end{array}$ & $\begin{array}{c}\text { Clean-catch } \\
\text { contaminated, } \\
n(\%)\end{array}$ & $\begin{array}{c}\text { Nappy pad } \\
\text { contaminated, } \\
n(\%)\end{array}$ & $\begin{array}{c}\text { Risk } \\
\text { difference, } \\
95 \% \mathrm{Cl}\end{array}$ & $\begin{array}{l}\text { Risk ratio, } \\
95 \% \mathrm{Cl}\end{array}$ & $P$-value \\
\hline $\begin{array}{l}\text { Jackson et al, } 2005^{11} \\
\text { frank contamination }\end{array}$ & $>10^{5}>2$ organisms & $\begin{array}{c}50 / 2740 \\
(1.8)\end{array}$ & $\begin{array}{l}277 / 2277 \\
(12.2)\end{array}$ & $\begin{array}{c}0.103 \\
\text { (0.089 to } 0.118)\end{array}$ & $\begin{array}{c}6.666 \\
(4.959 \text { to } 8.963)\end{array}$ & $<0.001$ \\
\hline $\begin{array}{l}\text { Feasey, } 1999^{13} \text { or Rao et al, } 2004^{12} \\
\text { heavy contamination }\end{array}$ & $>10^{5} \geq 2$ organisms & $\begin{array}{c}78 / 2740 \\
(2.8)\end{array}$ & $\begin{array}{c}426 / 2277 \\
(18.7)\end{array}$ & $\begin{array}{c}0.159 \\
(0.141 \text { to } 0.176)\end{array}$ & $\begin{array}{c}6.572 \\
\text { (5.196 to } 8.312)\end{array}$ & $<0.001$ \\
\hline $\begin{array}{l}\text { Jackson et al, } 2005^{11} \\
\text { or Bekeris et al, } 2008^{14} \\
\text { probable or frank contamination }\end{array}$ & $>10^{4}>2$ organisms & $\begin{array}{l}175 / 2740 \\
(6.4)\end{array}$ & $\begin{array}{c}599 / 2277 \\
(26.3)\end{array}$ & $\begin{array}{c}0.199 \\
(0.179 \text { to } 0.219)\end{array}$ & $\begin{array}{c}4.119 \\
\text { [3.513 to } 4.829 \text { ) }\end{array}$ & $<0.001$ \\
\hline
\end{tabular}

Frank contamination is the definition of contamination that was used in the subsequent analyses.

\section{Comparison of findings using nappy pads and catch samples}

The validated AUROC for the nappy pad model was inferior to the model derived using clean-catch samples, which was 0.87 for symptoms and signs, increasing to 0.90 with dipstick results (to be reported fully in a future publication). Table 3 provides the proportion of nappy pad and clean-catch samples considered to be contaminated according to three published definitions. ${ }^{11-14}$ 'Frankly contaminated' urine was found in $12.2 \%$ of nappy pad and $1.8 \%$ of clean-catch samples, risk ratio $6.66195 \% \mathrm{Cl}=4.95$ to 8.96; $P<0.001)$.

\section{DISCUSSION}

\section{Summary}

Four features (female sex, smelly urine, darker urine, and absence of nappy rash) that could be reported by parents, and no clinical signs, were associated with a microbiological diagnosis of UTI in children sampled using nappy pads. These features were substantially more predictive of a microbiological diagnosis of a UTI than clinicians' 'working' or clinical diagnoses, but less predictive than data obtained for older children sampled using the cleancatch methods. More than $10 \%$ of samples obtained by nappy pads were 'frankly' contaminated, compared with $<2 \%$ of samples obtained by clean-catch from predominantly older children. The addition of dipstick testing improved diagnostic accuracy of nappy pad samples.

\section{Strengths and limitations}

The DUTY study is the largest primary care diagnostic accuracy study of clinical symptoms, signs, and dipstick tests for diagnosing UTI in young children, and it achieved high levels of data completeness. Clinicians were asked to obtain a cleancatch urine sample whenever possible, but ultimately, the decision whether to sample by clean-catch or the nappy pad method was up to the parents, who generally used the nappy pad method in younger children. Children sampled using the nappy pad method were therefore younger and may have differed in other ways as well, for example being more unwell or distressed. Parents were asked to replace pads at regular intervals until a sample was obtained. However, alarms were not used to trigger a scheduled replacement of pad, and this may have led to increased contamination rates. ${ }^{12}$

There was a relatively small number of UTIs diagnosed microbiologically in this study, with fewer diagnosed from the fraction of the urine samples sent to the research laboratory compared to the fraction of the samples sent to the NHS laboratories, perhaps because of more intensive methods used in the research laboratory resulting in fewer falsepositives. It is plausible that the nappy pad contamination masked the presence of UTI leading to underdiagnosis in comparison with clean-catch samples, in which lower contamination and higher UTI rates were observed. The conservative criteria for a microbiological UTI diagnosis may have also contributed.

This study reference standard defined 'uropathogens' as members of the Enterobacteriaceae family at the UK guidelines' ${ }^{19,20}$ threshold of a pure/ predominant growth of $\geq 10^{5} \mathrm{CFU} / \mathrm{mL}$. It was decided not to use a lower threshold, as this carries an increased risk of false-positives, although there are recommendations that a lower threshold should be used., 3,21,22 The diagnosis of UTI is a clinical one, taking microbiological analysis into account, and a lower microbiological threshold in the presence of high clinical suspicion would be acceptable for the purposes of clinical care 
as opposed to this diagnostic study. This study used a rigorous criterion (minimum 3-log difference between the predominant and next most concentrated organism] for defining predominance. This definition could have reduced estimated prevalence if some UTIs were incorrectly classified as contamination. In addition, a small proportion of the positive cultures may have been false-positives due to asymptomatic bacteriuria or contamination.

Collecting an uncontaminated urine specimen is most difficult in the youngest children, and no method has yet been found to reliably distinguish pathogen from contaminant, especially when they coexist. The study's definition of UTI excluded atypical bacteria causing UTIs, which are also thought to be more common in younger children, potentially reducing this estimated UTI prevalence. . $^{1923}$

\section{Comparison with existing literature}

The NICE guidelines found insufficient data to draw conclusions about urine collection bags and urine collection pads', but recommended their use when a cleancatch sample cannot be obtained. ${ }^{3}$ The authors have been unable to identify further studies addressing nappy pads since the NICE recommendations were published. ${ }^{24}$ A systematic review of the accuracy of specimens obtained from nappy pads included three studies that compared sampling by nappy pad to sampling by urine bag, and one study that compared nappy pad specimens to specimens obtained by suprapubic aspiration. The latter study found $100 \%$ sensitivity and $94 \%$ specificity between the two methods. A randomised trial found that replacing pads every 30 minutes until a sample was obtained, reduced contamination. ${ }^{12}$

The authors found a $1.3 \%$ prevalence of microbiological diagnoses of UTI. The only other UK primary care study found a $6 \%$ prevalence when urine samples were analysed in NHS laboratories. ${ }^{2}$ A similar UTI prevalence of $5.6 \%$ for the DUTY study urine samples overall in NHS laboratories was found. ${ }^{18}$ Fever was not an inclusion criterion in that study or in the present study. However, a systematic review of 10 studies, eight of which were conducted in a hospital ED, with one in a clinic and ED setting, and one in a clinic setting, and all conducted in the US apart from a clinic study in Taiwan, found a UTI prevalence of $7 \%$ among infants presenting with fever. ${ }^{25}$ An Australian study (with incomplete urine sampling) found a prevalence of $3.4 \%$ children presenting with fever to EDs. ${ }^{26}$
The authors' systematic search identified one systematic review that included eight primary studies of 7892 children aged $<5$ years $^{24}$ and three further primary studies $^{26-28}$ that included 17462 children, that assessed associations between clinical features symptoms and signs and a UTI diagnosis. The data found showed that no individual symptom or sign or combination of symptoms or signs was sufficient to rule in a diagnosis of UTI. Among the remaining studies, largely conducted in hospital EDs, abdominal pain, back pain, dysuria, frequency, and new-onset urinary incontinence increased the likelihood of a UTI. ${ }^{29}$ Stridor, audible wheeze, circumcision, temperature $<39^{\circ} \mathrm{C}$ with a source, abnormal chest sounds, chest crackles, age $\leq 3$ years, not feeling hot, and breathing difficulty decreased the likelihood of UTIs. The largest study, which included almost 16000 children aged $<5$ years presenting to EDs in Australia, ${ }^{26}$ derived a diagnostic model based on a combination of 27 symptoms and signs. However, this study did not involve systematic urine sampling, and most children did not have urine sampled. This model was found to have an AUROC of $0.80(95 \% \mathrm{Cl}=0.78$ to 0.82$)$, which is similar to findings from this DUTY study for children sampled with nappy pads.

Previous investigation of malodorous urine has shown conflicting results, ${ }^{30}$ but the present study strongly supports its diagnostic value. The authors investigated, but did not find evidence for, a number of non-specific symptoms lincluding fever, vomiting, lethargy, irritability, and poor feeding) previously found to be associated with $U T^{24}$ and recommended for clinical use by NICE. ${ }^{3}$ It remains possible that such symptoms are of use in the secondary care settings in which studies reporting their utility were conducted, or in children with a different illness spectrum. This finding underlines the importance of including a wide range of illness presentation in studies of predictors of diagnoses, especially when symptoms and signs are notoriously nonspecific. Studies that include only children with symptoms and signs previously found to be associated with the diagnosis risks missing previously unidentified predictors and 'research circularity' llooking for, and finding, symptoms and signs in children included in studies if they have those symptoms and signs).

The reduction in the risk of UTI associated with presence of a nappy rash should be interpreted with caution. The inverse association may arise through lower likelihood of a UTI when there is a 


\section{Funding}

This project was funded by the National Institute for Health Research (NIHR) Health Technology Assessment (HTA) programme (project number 08/66/01) and will be published in full in Health Technology Assessment, July 2016. Further information is available at: www.DUTYstudy.org.uk. This report presents independent research commissioned by the National Institute for Health Research (NIHR). The views and opinions expressed by the authors in this publication are those of the authors, and do not necessarily reflect those of the NHS, the NIHR, Medical Research Council, Central Commissioning Facility, NIHR Evaluation Trials and Studies, the NIHR HTA programme, or the Department of Health.

\section{Ethical approval}

Multicentre ethical approval was granted by the South West Southmead Research Ethics Committee Ipreviously Southmead Research Ethics Committee, then South West 4 REC) (Ref \#09/H0102/64).

\section{Provenance}

Freely submitted; externally peer reviewed.

\section{Competing interests}

The authors have declared no competing interests.

\section{Acknowledgements}

The authors thank the children, their families, the GP practices and other recruitment sites, the research networks, the NHS laboratories, and all the members of the DUTY team. Thanks also to the providers of nursing and clinical studies officer support from the Primary Care Research Networks in: Greater London; Kent and Medway; Sussex; Surrey; Thames Valley; the South-west; Cumbria and Lancashire; Northumberland and Tyne and Wear; and the National Institute for Social Care and Health Research, Clinical Research Centre in Wales (NISCHR-CRC). Additional acknowledgement to the support given by the South East Wales Trials Unit (funded by NISCHR), Comprehensive Local Research Networks of Central and East London, Western, Peninsula, Hampshire and Isle of Wight, and the NIHR Biomedical Research and Development Department, Guy's and St Thomas' NHS Foundation Trust.

\section{Discuss this article}

Contribute and read comments about this article: bjgp.org/letters plausible alternative diagnosis /conditioning on the common effect of primary care attendance). ${ }^{31}$ Alternative explanations are that rash may be a risk factor for contamination of urine, and this masks the presence of a UTI, or that skin products used to treat nappy rash could render the urine sterile. However, the authors found no evidence of an association between nappy rash and contamination, nor the presence of antimicrobial substances in the urine and nappy rash. An increased likelihood of contamination of nappy pad samples could also explain the more modest associations of symptoms and dipstick test results with UTI than were found in clean-catch samples. ${ }^{10}$ The models were re-run excluding nappy rash and modest reductions were found in the symptoms and signs, and symptoms, signs, and dipstick model AUROCs (0.07 and 0.03, respectively).

\section{Implications for research and practice}

Nappy pad urine sample culture results, together with symptoms that can be reported by parents can be clinically useful in identifying acutely unwell pre-school children presenting to primary care who have a UTI, but with less accuracy, and with increased contamination compared with clean-catch sampling. Clean-catch urine sampling in children in primary care should be prioritised, especially in children with nappy rash. However, if sampling is done using nappy pads, then the addition of dipstick testing significantly improves diagnostic accuracy.

Further research is needed to distinguish pathogens from contaminants, and to establish the cost-effectiveness of different sensitivity and specificity cut points using routine health service laboratory results. It is not known precisely how results from clean-catch sampling compare with nappy pad sampling in younger children; contamination may vary by age as well as by sampling method. Randomising children to the sampling method could shed light on this. 


\section{REFERENCES}

1. Coulthard MG, Vernon SJ, Lambert HJ, et al. A nurse led education and direct access service for the management of urinary tract infections in children: prospective controlled trial. BMJ 2003; 327(7416): 656 .

2. O'Brien K, Edwards A, Hood K, Butler CC. Prevalence of urinary tract infection in acutely unwell children in general practice: a prospective study with systematic urine sampling. Br J Gen Pract 2013; DOI: 10.3399/bjgp13X663127.

3. National Institute for Health and Care Excellence. Urinary tract infection in children: diagnosis, treatment and long-term management. NICE Clinical Guideline 54. 2007. https://www.nice.org.uk/Guidance/CG54 laccessed 9 Jun 2016).

4. van der Voort J, Edwards A, Roberts R, et al. The struggle to diagnose UTI in children under two in primary care. Fam Pract 1997; 14(1): 44-48.

5. Liaw LC, Nayar DM, Pedler SJ, et al. Home collection of urine for culture from infants by three methods: survey of parents' preferences and bacterial contamination rates. BMJ 2000; 320(7245): 1312-1313.

6. Vernon S, Foo CK, Coulthard MG. How general practitioners manage children with urinary tract infection: an audit in the former Northern Region. Br J Gen Pract 1997; 47(418): 297-300.

7. Hay AD, Sterne AC, Hood K, et al. Improving the diagnosis and treatment of urinary tract infection in young children in primary care: results from the 'DUTY' prospective diagnostic cohort study. Ann Fam Med 2016; in press.

8. Downing H, Thomas-Jones $\mathrm{E}, \mathrm{Gal} \mathrm{M}$, et al. The diagnosis of urinary tract infections in young children (DUTY): protocol for a diagnostic and prospective observational study to derive and validate a clinical algorithm for the diagnosis of UTI in children presenting to primary care with an acute illness. BMC Infect Dis 2012; 12: 158.

9. Vernon S, Redfearn A, Pedler SJ, et al. Urine collection on sanitary towels. Lancet 1994; 344(8922): 612.

10. Hay AD, Birnie K, Busby J, et al. The diagnosis of urinary tract infections in young children (DUTY): a diagnostic and prospective observational study to derive and validate a clinical algorithm for the diagnosis of UTI in children presenting to primary care with an acute illness. Health Technol Assess 2016; in press.

11. Jackson SR, Dryden M, Gillett P, et al. A novel midstream urine-collection device reduces contamination rates in urine cultures amongst women. BJU Int 2005; 96(3): 360-364

12. Rao S, Bhatt J, Houghton C, et al. An improved urine collection pad method: a randomised clinical trial. Arch Dis Child 2004; 89(8): 773-775.

13. Feasey S. Are Newcastle urine collection pads suitable as a means of collecting specimens from infants? Paediatr Nurs 1999; 11(9): 17-21.

14. Bekeris LG, Jones BA, Walsh MK, et al. Urine culture contamination: a College of American Pathologists Q-Probes study of 127 laboratories. Arch Pathol Lab Med 2008; 132(6): 913-917.
15. Kinlin LM, Kirchner $\mathrm{C}$, Zhang $\mathrm{H}$, et al. Derivation and validation of a clinical prediction rule for nosocomial pneumonia after coronary artery bypass graft surgery. Clin Infect Dis 2010; 50(4): 493-501.

16. Rubin D. Inference and missing data. Biometrika 1976; 63(3): 581-592.

17. Steyerberg EW, Harrell FE Jr, Borsboom GJ, et al. Internal validation of predictive models: efficiency of some procedures for logistic regression analysis. J Clin Epidemiol 2001; 54(8): 774-781.

18. Butler CC, O'Brien K, Pickles T, et al. Childhood urinary tract infection in primary care: a prospective observational study of prevalence, diagnosis, treatment, and recovery. Br J Gen Pract 2015; DOI: 10.3399/bjgp15X684361.

19. Friedman S, Reif S, Assia A, et al. Clinical and laboratory characteristics of nonE. coli urinary tract infections. Arch Dis Child 2006; 91(10): 845-846.

20. Health Protection Agency. National standard method: investigation of urine. 2009; Report No. BSOP 41. https:/www.gov.uk/government/uploads/system/ uploads/attachment_data/file/458681/B_41i7.2.pdf (accessed 9 Jun 2016).

21. Grabe M, Bjerklund-Johansen TE, Botto $\mathrm{H}$, et al. Guidelines on urological infections. European Association of Urology 2013. http://uroweb.org/wp-content/ uploads/18_Urological-infections_LR.pdf laccessed 12 Mar 2016).

22. Scottish Intercollegiate Guidelines Network. Management of suspected bacterial urinary tract infection in adults. SIGN publication no.88. 2012. http:// www.sign.ac.uk/pdf/sign88.pdf laccessed 7 Jun 2016).

23. Paschke AA, Zaoutis T, Conway PH, et al. Previous antimicrobial exposure is associated with drug-resistant urinary tract infections in children. Pediatrics 2010; 125(4): 664-672.

24. Hay AD, Whiting $P$, Butler $C C$. How best to diagnose urinary tract infection in preschool children in primary care? BMJ 2011; 343: d6316.

25. Shaikh N, Morone NE, Bost JE, et al. Prevalence of urinary tract infection in childhood: a meta-analysis. Pediatr Infect Dis J 2008; 27(4): 302-308.

26. Craig JC, Williams GJ, Jones $M$, et al. The accuracy of clinical symptoms and signs for the diagnosis of serious bacterial infection in young febrile children: prospective cohort study of 15781 febrile illnesses. BMJ 2010; 340: c1594.

27. Gorelick MH, Shaw KN. Clinical decision rule to identify febrile young girls at risk for urinary tract infection. Arch Pediatr Adolesc Med 2000; 154(4): 386-390.

28. Armengol CE, Hendley JO, Schlager TA. Should we abandon standard microscopy when screening for urinary tract infections in young children? Pediatr Infect Dis J 2001; 20(12): 1176-1177.

29. Shaikh N, Morone NE, Lopez J, et al. Does this child have a urinary tract infection? JAMA: JAMA 2007; 298(24): 2895-2904.

30. Shaw KN, Hexter D, McGowan KL, et al. Clinical evaluation of a rapid screening test for urinary tract infections in children. J Pediatr 1991; 118(5): 733-736.

31. Lejeune B, Baron R, Guillois B, et al. Evaluation of a screening test for detecting urinary tract infection in newborns and infants. J Clin Pathol 1991; 44(12): 1029-1030 